\title{
Effects of Latency on Telesurgery: An Experimental Study
}

\author{
Reiza Rayman ${ }^{1}$, Serguei Primak ${ }^{2}$, Rajni Patel ${ }^{2}$, Merhdad Moallem², \\ Roya Morady ${ }^{1}$, Mahdi Tavakoli ${ }^{2}$, Vanja Subotic ${ }^{2}$, Natalie Galbraith ${ }^{2}$, \\ Aimee van Wynsberghe ${ }^{1}$, and Kris Croome $^{1}$ \\ 1 Canadian Surgical Technologies \& Advanced Robotics (CSTAR), \\ 339 Windermere Road, London, Ontario, N6A 5A5, Canada \\ 2 CSTAR \& Department of Electrical and Computer Engineering, \\ The University of Western Ontario, London, Ontario, N6A 5B9, Canada
}

\begin{abstract}
The paper is concerned with determining the feasibility of performing telesurgery over long communication links. It describes an experimental testbed for telesurgery that is currently available in our laboratory. The tesbed is capable of supporting both wired and satellite connections as well as simulated network environments. The feasibility of performing telesurgery over a satellite link with approximately $600 \mathrm{~ms}$ delay is shown through a number of dry and wet lab experiments. Quantative results of these experiments are also discussed.
\end{abstract}

\section{Introduction}

The use of minimally invasive surgical systems for performing remote surgery (telesurgery) has the potential to significantly improve healthcare in remote communities and provide cost effective services. While robot-assisted on-site minimally invasive surgery (MIS) has became a routine procedure, there has been very little experience gained so far in remote teleoperations of robotic MIS systems [1]-[5]. Our aboratory is currently developing and testing new infrastracture to investigate the feasibility of telesurgery over wired and satellite communication links. This work concentrates on the following issues: 1) development of an appropriate testbed; 2) dry lab experiments to evaluate the effects of communication latency; 3 ) determination of tasks that are appropriate for telesurgery; 4) wet lab experiments in the presence of different amounts of latency; 5) determination of the type and amount of training required for telesurgery.

It is well known that delays and disturbances in communication links can severely degrade the quality of teleoperation. However even approximate limits on such operations are currently not known. The experiments conducted in our Telesurgery Research Laboratory at CSTAR are aimed at addressing some of the issues related to telesurgery over large distances. In this paper, we present results that provide a quantitative evaluation of the effect of latency on the performance of telesurgery using both dry lab and wet lab (animal) experiments. 


\section{Experimental Setup}

The experimental setup consists of the following two major units: a teleoperation capable ZEUS ${ }^{T M}$ robotic MIS system [1 with both the surgeon's console and the surgical robot located in our laboratory and a communication network with its monitoring equipment.

A redundant dedicated communication link consisting of three different modalities has been established with the help of Bell Canada and Telesat Canada. These include a wired link via Halifax with a roundtrip delay of $64 \mathrm{~ms}$, a satellite link with a roundtrip delay of $580 \mathrm{~ms}$, and a software simulated delay link through a local switch. The network design provides considerable flexibility for expansion and redeployment, sufficient redundancy and graceful degradation in case of communication failures. The setup has been found to be suitable for both dry and wet lab experiments as described below.

\subsection{Telesurgery Unit}

The ZEUS ${ }^{T M}$ robotic MIS system has been designed using a master-slave philosophy as described in [1] with the master (surgeon's console) and the slave (surgical robot located in the operating room) comprising completely separate units that are both IP capable. The surgical robot at the patient side contains three robotic arms, two of which are used to manipulate laparoscopic surgical intruments while the third holds an endoscopic camera. The latter provides a 2D view of the surgical area inside the patient. All three arms are controlled from the surgeon's console. The arms are controlled remotely through a sequence of packet-oriented digital commands from the surgeon's console, and are delivered through a standard 100 base T ethernet network connection. The surgeon's console has two 5 degrees-of-freedom "arms" that are used by the surgeon to manipulate the two robotic arms at the patient side that are connected to laparoscopic surgical tools. The endoscopic camera unit at the patient side is controlled from the surgeon's console. The surgeon also has a view of a video stream provided by a color video camera located at the patient side.

An additional unit described in [1] allows the telesurgery system to operate in UDP/IP mode thus avoiding time-outs due to large delays. The unit is designed to lock the operation of the robot if the packet loss in the communication network becomes unacceptably high.

The video signal from the endoscopic camera in compressed by an encoder to preserve bandwidth. The encoder introduces a $100 \mathrm{~ms}$ processing delay. The image is restored into the original video format at the surgeon side by means of a decoder. An additional function of the encoder/decoder is to enable one to vary the effective transmission bandwidth utilized by the system. This is because most of the bandwidth resource is consumed by the videotraffic. Two sets of encoders and coders are used to provide redundancy. Both the surgeon and the patient sides are equipped with IP compatible Polycom ViewStations allowing lower quality video and audio interaction between the two sides. This link is also used to convey instructions from the surgeon to the remote side (such as turn on/off ultrasound scalpel, etc.). 


\subsection{Networking}

The general network configuration is shown in Fig. 1, All individual devices are connected to each other using a standard IP interface. This enables the system to be easily deployed, be readjusted or be augmented by new tools. Signals from all devices are collected and multiplexed by aswitch, creating a single data stream directed by a router. The networking hardware is fully redundant on both sides. The system automatically chooses between switches to obtain the most reliable link. The total bandwidth provided by Bell amounts to $10 \mathrm{Mbps}$ which is sufficient for transmitting all of the necessary signals with a high level of priority. Routers on each side can be connected in different ways to emulate different modalities of the deployment. The first possibility it to connect through a dedicated computer equipped with the NetDisturb software [2. This modality can be used for the purpose of training and testing of the system under a great variety of simulated conditions. The software allows one to isolate particular network parameters to study their influence on the setup performance. The second option includes a wired high quality, low latency (approximately $65 \mathrm{~ms}$ round trip)connection which loops from London to Halifax and back. Coupled with the delay in the codecs, this is roughly equivalent to a $200 \mathrm{~ms}$ round trip delay. Finally, a Telesat satellite link can also be included in the loop, providing a link with a variable time delay of approximately $600 \mathrm{~ms}$ round trip with some jitter and packet losses. Inclusion of such a segment is a substantial contribution since no such experiments have been reported in the literature. A protocol of switching between the wired and satellite loops has been agreed upon between the involved parties and the switching itself has been routinely used during actual experimentation.

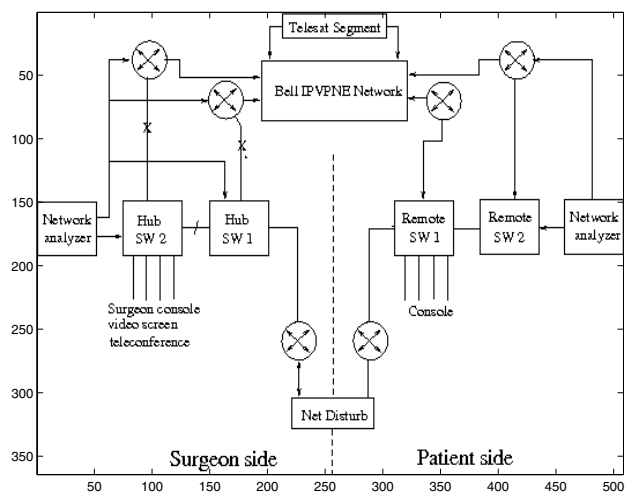

Fig. 1. Network configuration

\section{$2.3 \quad$ Experiments}

We have conducted numerous experiments to evaluate the performance of the system. In contrast to previously reported feasibility experiments [1], [3]-6, we 
have conducted a number of trials that provide more quantative information about such important parameters as completion time and quality of surgery. In this paper, we focus our attention on the dry lab experiments while briefly reporting the results of the wet lab experiments which are still ongoing and will be reported in detail at another time.

\subsection{Dry Lab Experiments}

The robotic exercises were designed to simulate typical surgical maneuvers. These involved object grasping and precise placement, object steering, and curved needle manipulation using the laparoscopic tools manipulated by the robot arms. We did not simulate more complex tasks such as knot tying or precise suture placement. Due to the technical limitations of the ZEUS robotic system, such complex tasks are quite difficult to complete even without any latency thus resulting in a very 'noisy' data set. The following exercises were evaluated:

1. Pick up a cone with the left hand. Place in a circle. Return the cone to its original position with the right hand. Pass it back and forth six times. Repeat this procedure four times for one data set.

2. Pick up a 6-0 suturing needle with the left hand. Maneuver the needle to enter at the left dot and exit from right dot. Retrieve the exiting needle with the right hand. Hand over the needle to the left hand and repeat six times. Repeat this procedure four times for one data set.

3. Pick up a ring with the left hand, grasping the ring at the black line. Maneuver the ring with both hands so that the right hand only holds it at the black line. Do not drop or let the ring touch the surface during the maneuver. Pass the ring back and forth four times. Repeat this procedure eight times for one data set.

4. Pick up a rod with the left hand. Pass the rod through three hoops without touching the hoops. Pass it back and forth through the hoops four times. Repeat this procedure three times for one data set;

Four test subjects with no previus experience (students with science backgrounds) were assigned to complete the surgical exercises. These subjects had dayly access to the robotic system and has performed the exercises five days per week over a period of four months. Therefore, they were continuously exposed to the system and had the opportunity to learn and adapt to the system.

The group performed the surgical exercises at latencies from 0 to $1 \mathrm{~s}$, in increments of $100 \mathrm{~ms}$. At each latency, each subject completed four data sets for each exercise. Additionally, the subjects performed the exercises with random delays between 0 to $1 s$ at the end of the training period. Task completion times and error rate were recorded for all exercises.

The delay in the network was controlled by the NetDisturb software. For each delay, the completion time and a number tally of errors for the maneuver were recorderd and later plotted as a function of delay. It was observed that a relatively small number of repetitions of the same maneuver led to a reduction of task completion times due to learning. It was also found that the effect of delay is not pronounced until the round trip time exceeds $400 \mathrm{~ms}$. 


\subsection{Wet Lab Experiments}

The wet lab experiments were designed to conduct an internal mammary artery (IMA) takedown on a pig using the ZEUS ${ }^{T M}$ Telesurgical System. The procedure was divided into two $45 \mathrm{~min}$. segments each using a different C-STAR network asset. The first segment used the Halifax loop (64 $\mathrm{ms}$ roundtrip delay) and the second used the satellite (537 $\mathrm{ms}$ round trip delay). Following each segment, the length of the IMA dissected was measured and the surgeon's skills were assessed according to the "Objective Structured Assessment of Technical Skills" sliding scale. During the first segment, $3.5 \mathrm{~cm}$ of the IMA was dissected, and in the second segment, $4.5 \mathrm{~cm}$ of the IMA was dissected. The surgeon was able to perform at both latencies with fluid instrument movements, maximum efficiency and no inadvertent damage to the tissue.

\section{Experimental Results}

A few representative results of the dry lab experiments described above are shown in Fig. 2, It shows the completion time for different experiments as a function of the delay. The experiments were conducted by a group of inexperienced users who were asked to repeatedly conduct the experiments starting from a negligible delay and then with delay increasing by $100 \mathrm{~ms}$ for each new set of runs. Solid lines in Fig. 2 illustrates the effect that we call long-term learning. This type of learning has to do with improving performance through repeating a task a number of times. The effect of long term learning remains with the participant over the whole period of experiments (just over three months). The gradual decline in completion time upto about $300 \mathrm{~ms}$ delay can thus be explained by this long-time learning. This figure also indicates that the minimum amount of training needed to properly perform simple tasks can be achieved over relatively short periods of time, equivalent to 3-4 weeks of one hour a day, three times a week training period.

This point can be further verified by comparing the results with incremental and random delay distribution. The latter was performed under random delays that were unknown to the users. The experiments were conducted after all the users had gained significant experience in manipulating the robot thereby reducing the effect of any long-time learning. The curves clearly show that delays of upto $300 \mathrm{~ms}$ do not have any significant impact on the performance of teleoperation tasks. Further increase in the delay gradually degrades the performance. However, one can conclude that it is possible to perform simple tasks with delays as high as $800 \mathrm{~ms}$ with a high level of accuracy. Overall it can be concluded that it is possible to perform basic surgical tasks in a simulated environment with delays as large as $800 \mathrm{~ms}$ with moderate training.

The results shown in Fig. 3 illustrate the effect we refer to as short-term learning. This phenomenon was observed experiments with random delays that are unknown to the operator. Each task was performed a number of times and it can be seen from the figure that the perfomance improved significantly at the second attempt. This indicates that the operator adapted to a particular delay 


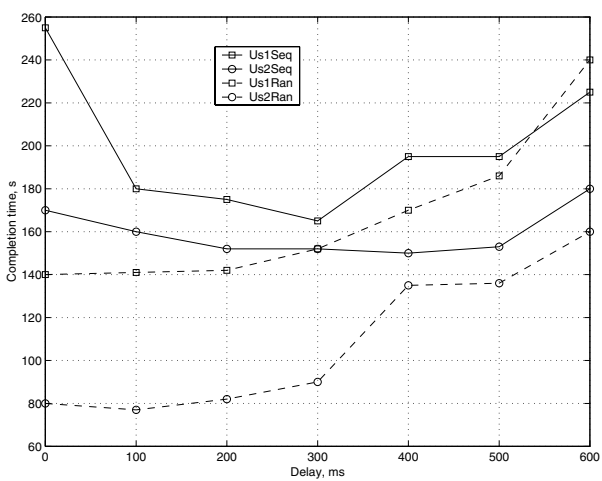

Fig. 2. Dependence of the completion time on the delay. The delay was gradually increased from $0 \mathrm{~ms}$ to $600 \mathrm{~ms}$ with $100 \mathrm{~ms}$ increments solid lines, or chosen at random, dashed lines.

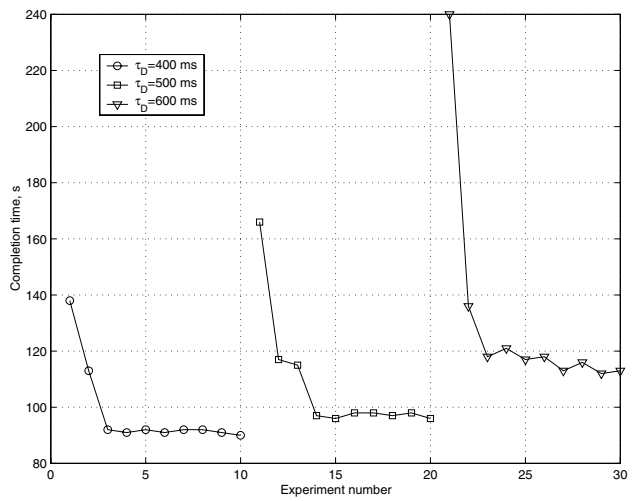

Fig. 3. Short term adaptation to a random delay

and his/her performance is optimized for this delay. However, once the delay changes, the skills acquired are no longer applicable and the operator has to adapt to a new delay. The skills acquired are therefore helpful only for a short period of time.

\section{Heuristic Mathematical Model}

While one cannot expect that a simple and accurate mathematical description of telesurgery can be developed, it is reasonable to explore the applicability of some basic models, based on Fitts' law 9] or feedback control.

The crossover model [7]-8] with one pole the open loop transfer function: $G(s)=\omega_{c} / s \exp \left(-s \tau_{D}\right)$ and the unity feedback is widely accepted as a model for a human operated control system. In essence, this is the simplest possible model which reflects the following fundamental properties of a human operator: 
Decision is based on some sort of information feedback; the human operator is able to react only to relatively slow changes in stimuli limited by the so-called crossover frequency $\omega_{c}$, and there is some delay $\tau_{D}$ between an observation and the reaction of the motor system.

In the absence of the delay $\tau_{D}=0$, the model gives a simple estimate of the task completion time for moving an object over a distance $A$ and placing it onto a target of width $W: T_{c}=1 / \omega_{c} \ln (2 A / W)$ if the task can be considered complete when the position of the target is in the range $A \pm 0.5 W$. This result agrees with Fitts' law 9. In the presence of a delay, one can distinguish three different regimes of operation: for relatively small delays, the exponential approach to the target remains, albeit the time of completion may be reduced ; for intermediate delays the system maintains stability; however, the approach to the target becomes oscillatory which, in turn, increases the completion time. For large delays the system becomes unstable or even chaotic [10].

While the model is simple and describes the main features concerning the human operator, it does not reflect such features as learning due to repeated practicing and adaptation to the delay during a single run of an experiment. We suggest the following generalization. The open-loop network is a series connection of a time-varying gain $K_{D}\left(t, \tau_{D}\right)$, a time-varying crossover frequency gain $1 / \tau_{h}\left(t, \tau_{D}\right)$, and the delay term. The variable gain represents short-term learning: decreasing the gain is equivalent to slowing the operation. It is assumed that at the initial stage of the movement, the human operator estimates the delay $\tau_{D}$ and adjusts the gain to avoid oscillatory movement. We suggest simple models of the dynamics of time delay estimation and gain adjustment as follows:

$$
\tau_{D}=\tau_{D 0}+\left(\tau_{D}^{*}-\tau_{D 0}\right) \exp \left(-\frac{t}{\tau_{a}}\right), K_{D}=K_{D 0}+\left(K_{D}^{*}-K_{D 0}\right) \exp \left(-\frac{t}{\tau_{a}}\right)
$$

This model reflects the fact that it takes a certain amount of time $\tau_{a}$ to acquire the necessary information about the delay, possible errors in the delay estimation, $\tau_{D}^{*} \neq \tau_{D}$, and that there is some initial guess about the delay in the network.

The variation in the parameter $\tau_{h}\left(\tau_{D}, N\right)$ reflects the long-term learning (i.e. learning over a number $N$ of repetitions of the same maneuver). During one run of the experiment, it remains constant but from run to run it may change due to a training process. It is reasonable to assume that the time needed to complete a task decreases from some level $\tau_{h 0}$ to a level of the best possible performance $\tau_{h}^{*}$. It is suggested that the task completion time decreases according to a power law $N^{-\alpha}[7]$.

\section{Concluding Remarks}

Preliminary results of the experiments indicate that telesurgery over long communication links is possible. It has been found that the maximum tolerable delay is approximately $600 \mathrm{~ms}$. This allows operations to be performed over wired links that cover most of continental Canada as well as through a one hop satellite link which allows for a much wider coverage. While there was some degradation in 
task completion times, it was shown experimentally that this was within tolerable limits. It was shown that the completion time complies with the basic Fitts' law. The results also show that significant improvement in performance can be achieved with proper training. However, additional work is required to quantify the effect of learning and to determine the maximum tolerable delay. Furthermore, an investigation of the quality of the communication channel on performance also needs to be performed.

\section{Acknowledgements}

The Authors would like to thank the Government of the Province of Ontario, Canada, Natural Sciences and Engineering Research Council of Canada, Canadian Foundation for Innovation, Bell Canada, Telesat Canada, Intuitive Surgical Inc, CA, USA for financial and technical support of this project.

\section{References}

1. S. E. Butner and M. Ghodoussi, "Transforming a surgical robot for human telesurgery", IEEE Trans. Robotics 8 Automation, Vol. 19, No. 5, pp. 818-824, Oct. 2003.

2. ZTI-Telecom NetDisturb http://www.zti-telecom.com/fr/pages/netdisturb.htm

3. J. C. Rosser, N. Gabriel, B. Herman and M. Murayama, "Telementoring and teleproctoring", World J. Surg., Vol.25, pp. 1438-1448, 2001.

4. R. E. Link, P. G. Schulam and L. R. Kavoussi, "Telesurgery, Remote monitoring and assistance during laparoscopy", Urol. Clin. North Am, Vol. 28, pp. 177-188, 2001.

5. B. R. Lee, D. J. Png, L. Liew et al., "Laparoscopic telesurgery between the United States and Singapore," Ann Acad. Med. Singapore, Vol. 29, pp. 665-668, 2000.

6. W. K. Cheah, B. Lee, J.E. Lenzi, P. M. Goh, "Telesurgical laparoscopic cholecystectomy between two countries," Surg. Endosc., Vol. 14, pp. 1085, 2000.

7. D. McRuer, D. Graham, E. Krendel, and W. Reisener, "Human Pilot Dynamics in Compensatory Systems - Theory, Models, and Experiments with Controlled Elements and Forcing Function variations," AFFDL-TR-65-15, July, 1965.

8. D. McRuer, and D. Graham, "Manual Control of Single Loop Systems: Part I," J. Franklin Institute, Vol. 283, pp. 1-29, 1967.

9. P. Fitts, "The information capacity of the human motor system in controlling the amplitude of movement," J. of Experimental Psychology, vol. 47, 1954, pp. 381-391.

10. P. Tass, J. Kurths, M.G. Rosenblum, G. Guasti, and H. Hefter, "Non-linear tracking Systems with Delay," Physical Review E, Vol. 54, pp. R2224-R2227, 1996.

11. A. McFoulkes, and C. Miall, "Adaptation to Visual Feedback Delays in a Human manual Tracking Task," Experimental Brain Research, Vol. 131, pp. 101-110, 2000. 